\title{
Adversarial Feature Disentanglement for Long-Term Person Re-identification
}

\author{
Wanlu Xu${ }^{1}$, Hong Liu ${ }^{1 *}$, Wei Shi ${ }^{1}$, Ziling Miao ${ }^{1}$, Zhisheng Lu$^{1}$ and Feihu Chen ${ }^{2}$ \\ ${ }^{1}$ Key Laboratory of Machine Perception, Shenzhen Graduate School, Peking University, China \\ ${ }^{2}$ Department of Precision Instrument, Tsinghua University, China \\ \{xuwanlu, hongliu,pkusw, zilingmiao, zhisheng_lu\}@pku.edu.cn, cfh19@mails.tsinghua.edu.cn
}

\begin{abstract}
Most existing person re-identification methods are effective in short-term scenarios because of their appearance dependencies. However, these methods may fail in long-term scenarios where people might change their clothes. To this end, we propose an adversarial feature disentanglement network (AFD-Net) which contains intra-class reconstruction and inter-class adversary to disentangle the identity-related and identity-unrelated (clothing) features. For intra-class reconstruction, the person images with the same identity are represented and disentangled into identity and clothing features by two separate encoders, and further reconstructed into original images to reduce intra-class feature variations. For inter-class adversary, the disentangled features across different identities are exchanged and recombined to generate adversarial clothes-changing images for training, which makes the identity and clothing features more independent. Especially, to supervise these new generated clothes-changing images, a re-feeding strategy is designed to re-disentangle and reconstruct these new images for image-level self-supervision in the original image space and feature-level soft-supervision in the disentangled feature space. Moreover, we collect a challenging Market-Clothes dataset and a real-world PKUMarket-Reid dataset for evaluation. The results on one large-scale short-term dataset (Market-1501) and five long-term datasets (three public and two we proposed) confirm the superiority of our method against other state-of-the-art methods.
\end{abstract}

\section{Introduction}

Person re-identification (Re-ID), the process of matching person images across different camera views, is widely used in person search [Shi et al., 2020] and multi-object tracking [Ke et al., 2019]. In recent years, many person Re-ID methods have made great success in short-term scenarios. These methods often rely heavily on the appearance of the people, which

\footnotetext{
${ }^{*}$ Contact Author
}

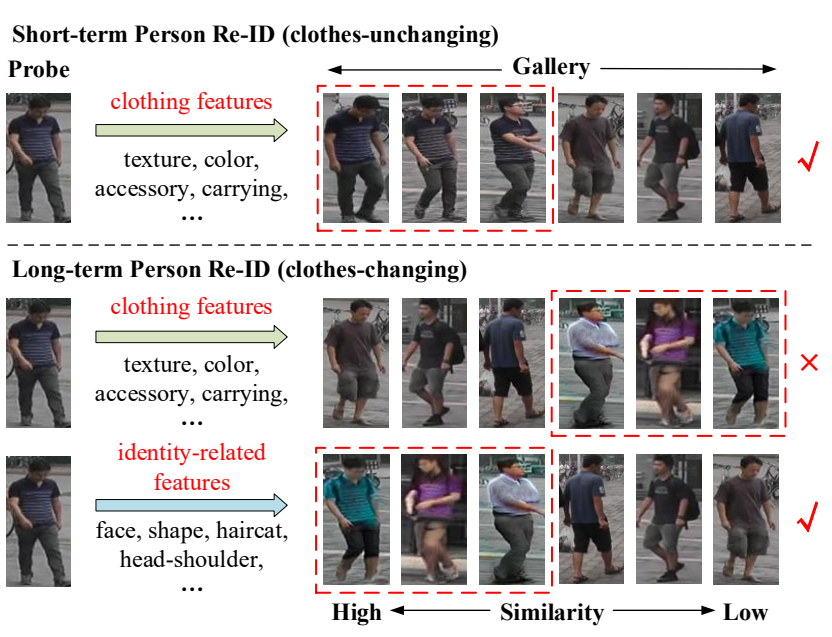

Figure 1: Examples of probe image and gallery candidates in the short-term and long-term scenarios from our generated dataset. The red dashed boxes represent the correct matching samples. Note that the identity-related features (face, shape, haircut, head-shoulder features, etc.) are more effective than clothing features (texture, color, accessory, carrying, etc.) to deal with clothes-changing problem.

makes them cannot be easily applied to address the clotheschanging problem in long-term scenarios. From Fig. 1, we find that the clothing features that are effective in short-term task become ineffective in long-term task, while identity features are discriminative to tackle the clothes-changing problem. Based on this observation, we aim to find how to split the identity and clothing information of person images and make them both independent and representative, so that our method can be adapted to both short and long-term scenarios.

Recently, great efforts have been devoted to extracting the robust identity representations of the human body for longterm person Re-ID task. Some works [Yang et al., 2020; Wan et al., 2020] proposed to use contour sketch or facial information as identity-related features for person reidentification. These methods only extract a certain type of explicit representation, but cannot get the complete identityrelated information of the human body. Other works [Li et al., 2020] proposed to use generated adversarial learning strategy to learn implicit shape-based features. These methods only pay attention to the adversarial learning between intra-class samples but ignore the important inter-class information. 
Considering the above problems, we propose an end-toend adversarial feature disentanglement network (AFD-Net) to disentangle identity-related and identity-unrelated (clothing) features. The AFD-Net combines intra-class reconstruction and inter-class adversary to make the two disentangled features independent and representative to adapt to different scenarios. At the same time, the inter-class network can recombine the identity and clothing information across different identities to obtain new adversarial clothes-changing images. Since there is no ground truth for generated images, a re-feeding strategy is designed to supervise the adversarial image generation. To be specific, the generated images are fed back to the AFD-Net to re-disentangle and reconstruct for image-level self-supervision in the original image space and feature-level soft-supervision in the disentangled feature space. Moreover, we collect two long-term datasets for evaluation. The Market-Clothes dataset is a generated challenging dataset in which each identity has 2 to 71 sets of clothes. The PKU-Market-Reid dataset is a collected real-world dataset, which has complex background and occlusion in a supermarket. It is worth mentioning that our method does not need to be trained on the clothes-changing dataset like many longterm methods. It can be used for long or short-term tasks only by training on short-term datasets such as Market-1501.

The main contributions can be summarized as follows:

- We propose an adversarial feature disentanglement network (AFD-Net) which contains intra-class reconstruction and inter-class adversary to disentangle the identityrelated and identity-unrelated (clothing) features. The inter-class network can recombine two features across identities to obtain adversarial clothes-changing images.

- To supervise the adversarial image generation, a refeeding strategy is designed to re-disentangle and reconstruct new generated images for image-level selfsupervision in the original image space and feature-level soft-supervision in the disentangled feature space.

- Two challenging datasets (Market-Clothes and PKUMarket-Reid) are collected for evaluation. The performance on one short-term dataset (Market-1501) and five long-term datasets (PRCC, Celeb-reID, Celeb-reIDlight, Market-Clothes, PKU-Market-Reid) confirm the effectiveness and generalizability of our method.

\section{Related Work}

Short-term person Re-ID. Short-term person Re-ID task is carried out based on the assumption that people do not change clothes. Most short-term methods focus on tackling the challenges of matching images under posture, viewpoint, background, illumination, or occlusion variations. We classify these methods into six categories: representation learning based methods [Geng et al., 2016], metric learning based methods [Varior et al., 2016], global features based methods [Zheng et al., 2017], local features based methods [Sun et al., 2018], video-based methods [Liu et al., 2018], and GAN-based methods [Zheng et al., 2019]. These methods can achieve high performance on many short-term datasets, but due to their appearance dependencies, they cannot be easily applied to address the clothes-changing problem.

\begin{tabular}{c|c|c|c|c|c}
\hline Dataset & Subjects & Images & Cameras & Clothes & Type \\
\hline Celeb-reID-light [Huang et al., 2020] & 590 & 10,842 & - & - & real \\
Celeb-reID [Huang et al., 2020] & 1,052 & 34,186 & - & - & real \\
PRCC [Yang et al., 2020] & 221 & 33,698 & 3 & - & real \\
LTCC [Qian et al., 2020] & 152 & 17,138 & 12 & $2-14$ & real \\
COCAS [Yu et al., 2020] & 5,266 & 62,382 & 30 & $2-3$ & real \\
Real28 [Wan et al., 2020] & 28 & 4,324 & 4 & 3 & real \\
PKU-Market-Reid (Ours) & $\mathbf{2 5}$ & $\mathbf{6 , 0 2 8}$ & $\mathbf{4}$ & $\mathbf{3 - 1 0}$ & real \\
\hline VC-Clothes [Wan et al., 2020] & 512 & 19,060 & 4 & $1-3$ & rendered \\
Div-Market [Li et al., 2020] & 200 & 24,732 & 6 & - & generated \\
Market-Clothes (Ours) & $\mathbf{7 5 1}$ & $\mathbf{1 6 , 4 8 3}$ & $\mathbf{6}$ & $\mathbf{2 - 7 1}$ & generated \\
\hline
\end{tabular}

Table 1: Comparison of various long-term person Re-ID datasets.

Long-term person Re-ID. People may change their clothes in long-term person Re-ID task, which makes longterm task face a huge challenge of appearance variations. Most of the current long-term person Re-ID methods aim at finding human representations that are independent of appearance information. These representations include global features fusing facial information [Wan et al., 2020], clothing features fusing biometric information [Yu et al., 2020], local features combining with attention mechanisms [Huang et al., 2020], and adversarial learning features [Li et al., 2020]. The first three representations belong to explicit feature learning, and the last one belongs to implicit feature learning. We believe that explicit learning can obtain exact body representations, while implicit learning is more likely to obtain discriminative features autonomously. Since the identity information in the long-term person Re-ID is critical and ambiguous, we aim to use the implicit learning method. [Zheng et al., 2019] proposed a joint learning method to couple Re-ID learning and data generation end-to-end. They think that the appearance information indicates the identity of the person, which is effective for short-term task. However, in our long-term task, it is more reasonable to regard identity as information independent of appearance. [Li et al., 2020] proposed a novel representation learning method that can generate a shape-based feature representation that is invariant to clothing. They only pay attention to the adversarial learning between intra-class samples but ignore the inter-class case. In our method, we simultaneously obtain intra-class and inter-class information to enhance the independence of identity and clothing features.

Long-term datasets. Due to the difficulty of collection, there are fewer long-term datasets. Huang et al. [Huang et al., 2020] built two large-scale long-term datasets called "CelebreID-light" and "Celeb-reID", which were acquired from the Internet using street snap-shots of celebrities. Yu et al. [Yu et al., 2020] constructed a novel large-scale Re-ID benchmark named clothes changing person set (COCAS), which combined a new Re-ID setting with clothes template. Yang et al. [Yang et al., 2020] and Qian et al. [Qian et al., 2020] proposed two real-world long-term datasets PRCC and LTCC respectively. Wan et al. [Wan et al., 2020] and Li et al. [Li et al., 2020] used generation or rendering methods to get two "fake" clothes-changing datasets. We choose the three largest datasets mentioned above to evaluate our method. And taking into account the high efficiency of the generation method and the practicability of the real scene, we also collect two longterm datasets: Market-Clothes and PKU-Market-Reid. The comparison of various long-term datasets is shown in Table 1. 


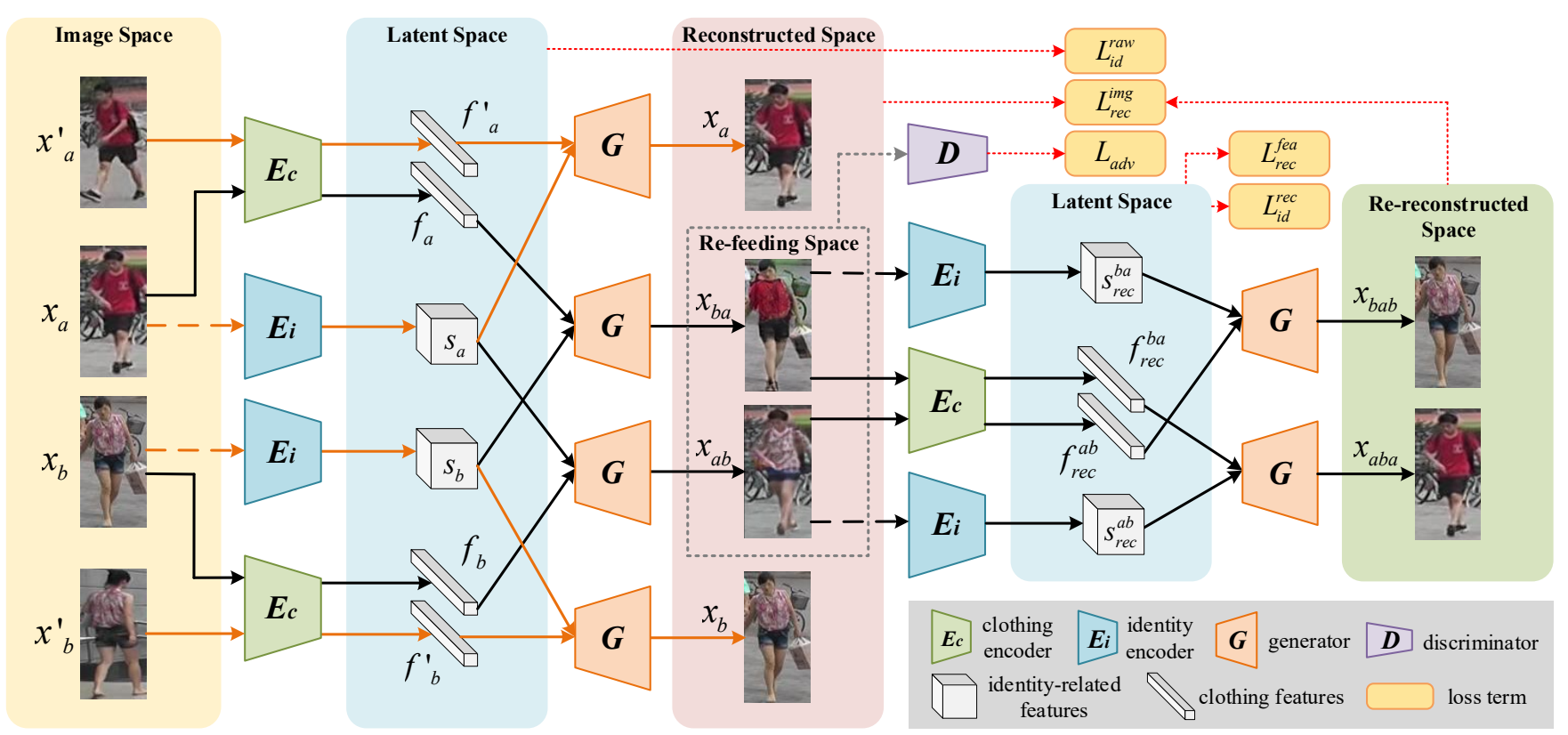

Figure 2: Overview of the proposed adversarial feature disentanglement network (AFD-Net). In disentanglement part, take $x_{a}$ as the target: (a) for intra-class, the identity and clothing encoders $E_{i}, E_{c}$ separately encode input images $x_{a}, x_{a}^{\prime}$ to produce identity-related and clothing features $s_{a}, f_{a}^{\prime}$. The generator $G$ jointly takes the $s_{a}$ and $f_{a}^{\prime}$ to reconstruct the image $x_{a}$. (b) For inter-class, $s_{a}$ and $f_{b}$ are obtained through the same encoders $E_{i}, E_{c}$ from $x_{a}, x_{b}$. The generator $G$ jointly takes the $s_{a}$ and $f_{b}$ to generate a new clothes-changing image $x_{a b}$. The discriminator $D$ is developed to determine whether the $x_{a b}$ and $x_{a}$ are from the same distribution. All operations are the same for $x_{b}$. In refeeding part: (c) the generated images $x_{a b}, x_{b a}$ are fed back to $E_{i}, E_{c}$ to reconstruct the original images $x_{a}, x_{b}$ for supervision of adversarial image generation. For better viewing, the dashed inputs of $E_{i}$ denote the gray images, the orange lines represent the intra-class operations.

\section{Proposed Method}

In this section, the proposed adversarial feature disentanglement network (AFD-Net) is illustrated in Fig. 2. The inputs of the AFD-Net are four images including two pairs from two identities. The intra-class image pair is disentangled and reconstructed into original images to reduce intra-class feature variations. The inter-class image pair is disentangled and recombined to generate new adversarial clothes-changing images. Moreover, a re-feeding strategy is designed to supervise the adversarial image generation by re-disentangling and reconstructing these new images, which contains image-level self-supervision in the original image space and feature-level soft-supervision in the disentangled feature space.

\subsection{Intra-Class Reconstruction}

The goal of this part is to encourage the identity and clothing encoders to pull their codes of the same identity together so that intra-class feature variations are reduced. Specifically, the encoders $E_{i}, E_{c}$ are used to separately extract identity and clothing features $s, f$ of two different images $x, x^{\prime}$ from the same person. Then the generator $G$ combines these two features to reconstruct the original image $x$ that provides identity features before. The image-level reconstruction loss $L_{\text {recon }}^{i m g}$ for $x_{a}, x_{b}$ is defined as:

$$
L_{\text {recon }}^{i m g}=\left\|x_{a}-G\left(s_{a}, f_{a}^{\prime}\right)\right\|_{1}+\left\|x_{b}-G\left(s_{b}, f_{b}^{\prime}\right)\right\|_{1},
$$

where $\|\cdot\|_{1}$ denotes the L1 norm. L1 norm is widely adopted in reconstruction loss since it can preserve image sharpness.
In the meantime, to utilize labeled information of training data for person re-ID, we employ classification loss on the identity and clothing features of person images. That can force these two features of different identities to stay apart. The classification loss for identity and clothing features are:

$$
\begin{aligned}
& L_{i d}^{s}=L_{i d}^{s_{a}}+L_{i d}^{s_{b}}=-\log \left(p\left(\hat{y}_{a} \mid x_{s_{a}}\right)\right)-\log \left(p\left(\hat{y}_{b} \mid x_{s_{b}}\right)\right), \\
& L_{i d}^{f^{\prime}}=L_{i d}^{f_{a}^{\prime}}+L_{i d}^{f_{b}^{\prime}}=-\log \left(p\left(\hat{y}_{a} \mid x_{f_{a}^{\prime}}\right)\right)-\log \left(p\left(\hat{y}_{b} \mid x_{f_{b}^{\prime}}\right)\right),
\end{aligned}
$$

where $p\left(\hat{y}_{a} \mid x_{s_{a}}\right)$ and $p\left(\hat{y}_{a} \mid x_{f_{a}^{\prime}}\right)$ are the predicted probabilities that $x_{a}$ and $x_{a}^{\prime}$ belong to the ground-truth class $\hat{y}_{a}$ separately based on their identity and clothing codes. The probabilities $p\left(\hat{y}_{b} \mid x_{s_{b}}\right)$ and $p\left(\hat{y}_{b} \mid x_{f_{b}^{\prime}}\right)$ have the same definition.

\subsection{Inter-Class Adversary}

After the intra-class feature variations are reduced, the interclass adversary is carried out across different identities, which can force the identity and clothing encoders to learn their respective relevant information. To be more precise, first, two encoders $E_{i}, E_{c}$ are used to separately extract identity and clothing features of two different identities. Second, the identity and clothing features are recombined to generate new adversarial clothes-changing images, like $x_{a b}=G\left(s_{a}, f_{b}\right)$ and $x_{b a}=G\left(s_{b}, f_{a}\right)$. Since there are no clothes-changing images in the same pose as the original images in the dataset, we cannot get the ground truth of the generated images. It means that the adversarial image generation has no pixel-level groundtruth supervision, so we introduce a re-feeding strategy for 
supervision later. To make features representational as intraclass, the classification loss is also employed on clothing features of $x_{a}$ and $x_{b}$ :

$$
L_{i d}^{f}=L_{i d}^{f_{a}}+L_{i d}^{f_{b}}=-\log \left(p\left(\hat{y}_{a} \mid x_{f_{a}}\right)\right)-\log \left(p\left(\hat{y}_{b} \mid x_{f_{b}}\right)\right),
$$

where $p\left(\hat{y}_{a} \mid x_{f_{a}}\right), p\left(\hat{y}_{b} \mid x_{f_{b}}\right)$ are the predicted probabilities that $x_{a}, x_{b}$ belong to the class $\hat{y}_{a}, \hat{y}_{b}$ based on clothing code.

The total classification loss of raw features $L_{i d}^{r a w}$ is:

$$
L_{i d}^{r a w}=L_{i d}^{s}+L_{i d}^{f}+L_{i d}^{f^{\prime}}
$$

To further enforce $G$ to perform content recovery, we produce perceptually realistic outputs by using the discriminator $D$ to discriminate the real images $x_{a}, x_{b}$ with the generated images $x_{a b}, x_{b a}$. The adversarial loss $L_{a d v}^{a b}, L_{a d v}^{b a}$ are:

$$
\begin{aligned}
& L_{a d v}^{a b}=\log \left(D\left(x_{a}\right)\right)+\log \left(1-D\left(x_{a b}\right)\right), \\
& L_{a d v}^{b a}=\log \left(D\left(x_{b}\right)\right)+\log \left(1-D\left(x_{b a}\right)\right) .
\end{aligned}
$$

The total adversarial loss $L_{a d v}$ is:

$$
L_{a d v}=L_{a d v}^{a b}+L_{a d v}^{b a} .
$$

\subsection{Image Re-feeding}

Since there is no ground truth for clothes-changing images, a re-feeding strategy is designed to supervise the adversarial image generation. The re-feeding strategy contains imagelevel self-supervision in the original image space and featurelevel soft-supervision in the disentangled feature space. For image-level self-supervision, the generated images are fed back to AFD-Net to re-disentangle and reconstruct into the original images. Like $x_{a b}, x_{b a}$ corresponding to $x_{a}, x_{b}$, we can get $x_{a b a}, x_{b a b}$ from $x_{a b}, x_{b a}$, which is formulated as:

$$
\begin{aligned}
& x_{a b a}=G\left(s_{r e c}^{a b}, f_{r e c}^{b a}\right), \\
& x_{b a b}=G\left(s_{r e c}^{b a}, f_{r e c}^{a b}\right),
\end{aligned}
$$

where the features $s_{r e c}^{a b}, f_{r e c}^{b a}$ derive from $x_{a}$, and the features $s_{r e c}^{b a}, f_{r e c}^{a b}$ derive from $x_{b}$. We find that the above-mentioned reconstructed images $x_{a b a}, x_{b a b}$ are exactly the original images $x_{a}, x_{b}$. Thus, we can achieve self-supervision by supervising the original images. The image-level reconstruction loss $L_{r e-r e c o n}^{i m g}$ in re-feeding part can be expressed as:

$$
L_{r e-r e c o n}^{i m g}=\left\|x_{a}-x_{a b a}\right\|_{1}+\left\|x_{b}-x_{b a b}\right\|_{1} .
$$

We add $L_{r e-r e c o n}^{i m g}$ and $L_{\text {recon }}^{i m g}$ calculated before to obtain the total image-level reconstruction loss $L_{r e c}^{i m g}$ :

$$
L_{r e c}^{i m g}=L_{\text {recon }}^{i m g}+L_{\text {re-recon }}^{i m g} .
$$

For feature-level soft-supervision, each generated image is softly supervised by two corresponding original images in the disentangled feature space. For example, the identity and clothing features of $x_{a b}$ are supervised by $x_{a}$ and $x_{b}$ respectively. The feature-level reconstruction loss $L_{r e c}^{s}, L_{r e c}^{f}$ are:

$$
\begin{aligned}
& L_{r e c}^{s}=\left\|s_{a}-s_{r e c}^{a b}\right\|_{1}+\left\|s_{b}-s_{r e c}^{b a}\right\|_{1}, \\
& L_{r e c}^{f}=\left\|f_{b}-f_{r e c}^{a b}\right\|_{1}+\left\|f_{a}-f_{r e c}^{b a}\right\|_{1},
\end{aligned}
$$

The total feature-level reconstruction loss $L_{\text {rec }}^{f e a}$ is:

$$
L_{r e c}^{f e a}=L_{r e c}^{s}+L_{r e c}^{f} .
$$

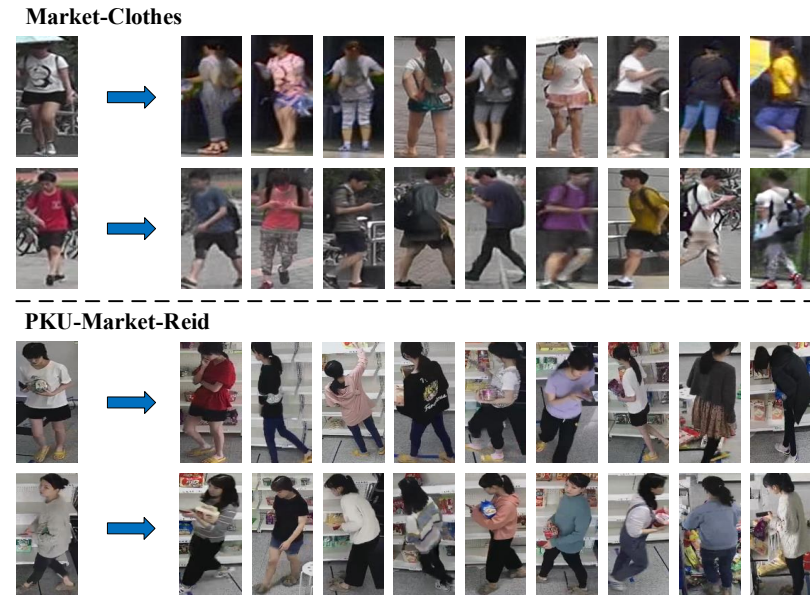

Figure 3: Examples of generated Market-Clothes and collected PKU-Market-Reid datasets. Each identity is shown in each row.

The classification loss is also employed on $s_{\text {rec }}$ and $f_{\text {rec }}$. Since the two reconstructed images $x_{a b}$ and $x_{b a}$ are generated from two images with different ID, how to determine their labels is an important problem. We introduce a soft label strategy to tackle this problem, and prove its effectiveness in ablation experiments. For example, because the image $x_{a b}$ is generated by features $s_{a}$ and $f_{b}$, we set the ground truth of $s_{r e c}^{a b}$ as $y_{a}$, of $f_{r e c}^{a b}$ as $y_{b}$ to be consistent with $s_{a}$ and $f_{b}$. This can further force the two encoders to output mutually independent features. The classification loss $L_{i d}^{r e s}, L_{i d}^{r e f}$ are:

$$
\begin{aligned}
& L_{i d}^{r e s}=L_{i d}^{s_{a b}}+L_{i d}^{s_{b a}}=-\log \left(p\left(\hat{y}_{a} \mid x_{s_{a b}}\right)\right)-\log \left(p\left(\hat{y}_{b} \mid x_{s_{b a}}\right)\right), \\
& L_{i d}^{r e f}=L_{i d}^{f_{a b}}+L_{i d}^{f_{b a}}=-\log \left(p\left(\hat{y}_{b} \mid x_{f_{a b}}\right)\right)-\log \left(p\left(\hat{y}_{a} \mid x_{f_{b a}}\right)\right),
\end{aligned}
$$

where $p\left(\hat{y}_{a} \mid x_{s_{a b}}\right)$ and $p\left(\hat{y}_{b} \mid x_{f_{a b}}\right)$ are the predicted probabilities that $x_{a b}$ belongs to the ground-truth class $\hat{y}_{a}$ and $\hat{y}_{b}$ separately based on its identity and clothing codes. The probabilities $p\left(\hat{y}_{b} \mid x_{s_{b a}}\right)$ and $p\left(\hat{y}_{a} \mid x_{f_{b a}}\right)$ have the same definition. The total classification loss of reconstructed features $L_{i d}^{r e c}$ is:

$$
L_{i d}^{r e c}=L_{i d}^{r e s}+L_{i d}^{r e f} .
$$

In summary, through the image reconstruction of $x_{a b a}$ and $x_{b a b}$ in image-level self-supervision, the feature reconstruction and classification of $s_{r e c}$ and $f_{r e c}$ in feature-level softsupervision, the adversarial image generation is supervised and new reliable clothes-changing images are generated.

\subsection{Optimization}

We train the AFD-Net end-to-end, and the sum of the abovementioned losses in the entire network is shown as:

$$
\begin{aligned}
L_{\text {total }} & =\lambda_{\text {rec }}^{i m g} \cdot L_{r e c}^{i m g}+\lambda_{r e c}^{f e a} \cdot L_{r e c}^{f e a}+\lambda_{a d v} \cdot L_{a d v} \\
& +\lambda_{i d}^{r a w} \cdot L_{i d}^{r a w}+\lambda_{i d}^{r e c} \cdot L_{i d}^{r e c}
\end{aligned}
$$

Following [Huang et al., 2018], we use a large weight $\lambda_{\text {rec }}^{i m g}=5$ for the image reconstruction loss. The feature reconstruction loss is inaccurate in the initial stage, so we gradually increase $\lambda_{\text {rec }}^{f e a}$ from 0 to 1 during the training process. Similarly, the identification loss $L_{i d}^{r e c}$ may make the training unstable due to the low quality of adversarial generated images at the beginning, so we set a small weight $\lambda_{i d}^{r e c}=0.5$. The remaining weights $\lambda_{i d}^{r a w}$ and $\lambda_{a d v}$ are set to 1 . 


\section{Experiments}

\subsection{Datasets}

We evaluate our method on six person Re-ID datasets, including three large-scale long-term datasets: Celeb-reID, Celeb-reID-light, PRCC, one benchmark short-term dataset: Market-1501, and two collected long-term datasets: MarketClothes, PKU-Market-Reid which are shown in Fig. 3.

Celeb-reID / Celeb-reID-light. Celeb-reID [Huang et al., 2020] is composed of 34,186 images of 1,052 identities that crawled from websites. It has 20,208 images from 632 identities for training and 13,978 images from 420 identities for testing. Celeb-reID-light is a light version of Celeb-reID, which has 490 and 100 identities for training and testing.

PRCC. PRCC [Yang et al., 2020] consists of 33,698 images from 221 identities. Each person in Cameras A and B is wearing the same clothes, and in $\mathrm{A}$ and $\mathrm{C}$ is wearing different clothes. The dataset has 150 and 71 identities for training and testing, and also provides contour sketch images for use.

Market-1501. Market-1501 [Zheng et al., 2015] is composed of 32,668 images of 1,501 identities collected from 6 camera views. The dataset is split into two non-overlapping fixed parts: 12,936 images from 751 identities for training and 19,732 images from 750 identities for testing.

Market-Clothes. Market-Clothes is our generated longterm dataset which is shown in Fig. 3. We change the clothes of person in Market-1501. It consists of 16,483 images from 751 identities, and each identity has 2 to 71 sets of clothes. It is a challenging dataset only used for the test phase.

PKU-Market-Reid. PKU-Market-Reid is our collected dataset which is shown in Fig. 3. It contains 6,028 images of 25 identities collected from 4 camera views, and each identity has 3 to 10 sets of clothes. This dataset contains different clothes, pose, illumination, shielding, and other complex conditions in supermarket. It is only used for the test phase too.

\subsection{Implementation Details}

We implement the AFD-Net using PyTorch with only one NVIDIA GTX 1080Ti GPU. All images are resized to $256 \times$ 128 for input. The SGD optimizer is used to train $E_{i}, E_{c}$ with $l r=0.002$, momentum $=0.9$. Encoders of the same type in AFD-Net share parameters. The Adam optimizer is applied to optimize $G, D$ with $l r=0.0001,(\beta 1, \beta 2)=(0,0.999)$. We use ResNet50 [He et al., 2016] pre-trained on ImageNet as our backbone of $E_{i}$ and $E_{c}$, and convert the input of $E_{i}$ to grayscale for color filtering. In the test phase, $E_{i}$ outputs the identity code in batch $\times 2048, E_{c}$ outputs the clothing code in batch $\times 1024$. We use the concatenation of two features for long-term task, and clothing features for short-term task.

\subsection{Comparison with State-of-the-Arts}

In this part, the common methods and long-term methods are split into two big rows, and the symbol $R$ is short for Rank.

Celeb-reID / Celeb-reID-light. Experimental results on two high-resolution long-term datasets are shown in Table 2. Our AFD-Net outperforms all the compared methods on Rank-1 and Rank-5 but underperforms the mAP of method

\begin{tabular}{c|ccc|ccc}
\hline \multirow{2}{*}{ Method } & \multicolumn{3}{|c|}{ Celeb-reID } & \multicolumn{3}{c}{ Celeb-reID-light } \\
\cline { 2 - 7 } & R1 & R5 & mAP & R1 & R5 & mAP \\
\hline MLFN [Chang et al., 2018] & 41.4 & 54.7 & 6.0 & 10.6 & 31.0 & 6.3 \\
IDE+DesNet121 [Zheng et al., 2017] & 42.9 & 56.4 & 5.9 & 10.5 & 24.8 & 5.3 \\
ResNet-Mid [Yu et al., 2017] & 43.3 & 54.6 & 5.8 & 10.3 & 28.0 & 6.0 \\
HACNN [Li et al., 2018] & 47.6 & 63.3 & 9.5 & 16.2 & 42.8 & 11.5 \\
MGN [Wang et al., 2018] & 49.0 & 64.9 & $\mathbf{1 0 . 8}$ & 21.5 & 47.4 & $\mathbf{1 3 . 9}$ \\
\hline ReIDCaps [Huang et al., 2020] & 51.2 & 65.4 & 9.8 & 20.3 & 48.2 & 11.2 \\
AFD-Net (Ours) & $\mathbf{5 2 . 1}$ & $\mathbf{6 6 . 1}$ & 10.6 & $\mathbf{2 2 . 2}$ & $\mathbf{5 1 . 0}$ & 11.3 \\
\hline
\end{tabular}

Table 2: Comparison on Cele-reID and Cele-reID-light datasets.

\begin{tabular}{c|ccc|ccc}
\hline \multirow{2}{*}{ Method } & \multicolumn{3}{|c|}{$\begin{array}{c}\text { Camera C to A } \\
\text { (Change clothes) }\end{array}$} & \multicolumn{3}{c}{$\begin{array}{c}\text { Camera B to A } \\
\text { (Same clothes) }\end{array}$} \\
\cline { 2 - 7 } & R1 & R10 & R20 & R1 & R10 & R20 \\
\hline Alexnet [Krizhevsky et al., 2012] & 16.3 & 48.0 & 65.9 & 63.3 & 91.7 & 94.7 \\
VGG16 [Simonyan and Zisserman, 2015] & 18.2 & 46.1 & 60.8 & 71.4 & 95.9 & 98.7 \\
Res-Net50 [He et al., 2016] & 19.4 & 52.4 & 66.4 & 74.8 & 97.3 & 98.9 \\
HACNN [Li et al., 2018] & 21.8 & 59.5 & 67.5 & 82.5 & 98.1 & 99.0 \\
PCB [Sun et al., 2018] & 22.9 & 61.2 & 78.3 & 86.9 & 98.8 & 99.6 \\
\hline Deformable Conv [Dai et al., 2017] & 26.0 & 71.7 & 85.3 & 61.9 & 92.1 & 97.7 \\
STN [Jaderberg et al., 2015] & 27.5 & 69.5 & 83.2 & 59.2 & 91.4 & 96.1 \\
SPT+ASE [Yang et al., 2020] & 34.4 & 77.3 & 88.1 & 64.2 & 92.6 & 96.7 \\
AFD-Net (Ours) & $\mathbf{4 2 . 8}$ & $\mathbf{7 9 . 6}$ & $\mathbf{8 9 . 0}$ & $\mathbf{9 5 . 7}$ & $\mathbf{9 9 . 0}$ & $\mathbf{9 9 . 8}$ \\
\hline
\end{tabular}

Table 3: Comparison on PRCC dataset.

\begin{tabular}{c|cccc}
\hline Method & R1 & R5 & R10 & mAP \\
\hline MLFN [Chang et al., 2018] & 90.0 & - & - & 74.3 \\
FD-GAN [Ge et al., 2018] & 90.5 & 96.0 & 97.7 & 77.9 \\
HACNN [Li et al., 2018] & 91.2 & - & - & 75.7 \\
PCB [Sun et al., 2018] & 93.2 & 97.3 & 98.2 & 81.7 \\
DG-Net [Zheng et al., 2019] & 94.4 & 98.4 & 98.9 & 85.2 \\
MGN [Wang et al., 2018] & $\mathbf{9 5 . 7}$ & - & - & $\mathbf{8 6 . 9}$ \\
\hline ReIDCaps [Huang et al., 2020] & 89.0 & - & - & 72.7 \\
ReIDCaps+ [Huang et al., 2020] & 92.8 & - & - & 78.0 \\
AFD-Net (Ours) & 94.2 & 98.0 & 98.7 & 84.3 \\
\hline
\end{tabular}

Table 4: Comparison on Market-1501 dataset.

\begin{tabular}{c|cccc|cccc}
\hline \multirow{2}{*}{ Method } & \multicolumn{4}{|c|}{ Market-Clothes } & \multicolumn{4}{c}{ PKU-Market-Reid } \\
\cline { 2 - 8 } & R1 & R5 & R10 & mAP & R1 & R5 & R10 & mAP \\
\hline FD-GAN [Ge et al., 2018] & 1.25 & 4.33 & 7.63 & 0.42 & 42.19 & 46.88 & 51.56 & 16.41 \\
Part-aligned [Suh et al., 2018] & 1.31 & 3.56 & 6.53 & 0.39 & 39.84 & 44.53 & 46.88 & 15.84 \\
PCB [Sun et al., 2018] & 2.82 & 8.14 & 12.90 & 0.71 & 45.66 & 48.78 & 55.03 & 16.65 \\
P2Net [Guo et al., 2019] & 8.60 & 15.62 & 23.44 & 5.70 & 43.16 & 47.66 & 52.34 & 14.68 \\
DG-Net [Zheng et al., 2019] & 20.04 & 33.09 & 39.64 & 6.32 & 44.53 & 49.78 & 53.91 & 17.30 \\
\hline ReIDCaps [Huang et al., 2020] & 15.52 & 22.59 & 27.64 & 6.60 & 43.91 & 46.69 & 50.81 & 15.66 \\
AFD-Net (Ours) & $\mathbf{4 8 . 3 1}$ & $\mathbf{6 5 . 8 8}$ & $\mathbf{7 2 . 7 7}$ & $\mathbf{8 . 4 7}$ & $\mathbf{4 6 . 8 8}$ & $\mathbf{5 0 . 0 2}$ & $\mathbf{5 6 . 2 5}$ & $\mathbf{1 8 . 0 5}$ \\
\hline
\end{tabular}

Table 5: Comparison on Market-Clothes and PKU-Market-Reid.
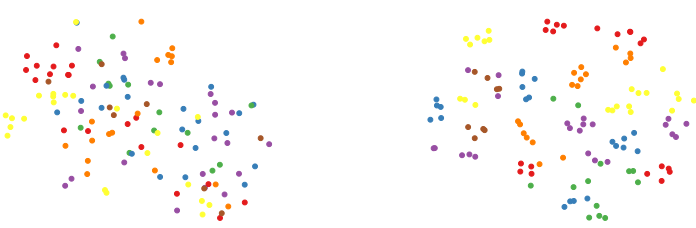

Figure 4: Visualization of identity features on Market-Clothes via t-SNE. We visualize 30 different identities by using ReIDCaps (left) and our AFD-Net (right), each of which is shown in a unique color.

MGN. The reason is that MGN utilizes multi-granularity features for Re-ID, which not only increases the mAP but also increases the complexity. However, our method can achieve comparable mAP by only using two disentangled features.

PRCC. As shown in Table 3, the Rank-1 / mAP of AFDNet achieves $42.8 \%$ / $89.0 \%$ in clothes-changing task, and 


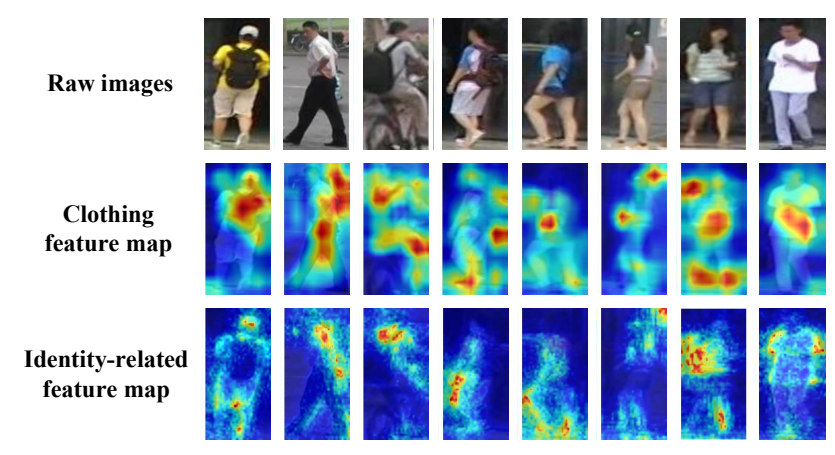

Figure 5: Visualization of clothing and identity feature maps on Market-1501 via AFD-Net. Each identity is shown in each column.

$95.7 \%$ / 99.8\% in clothes-unchanging task, which outperforms all the other methods by a large margin. This is because our approach can disentangle identity and clothing features, make them more independent to adapt to different scenarios.

Market-1501. To further prove that our method can also guarantee high performance in short-term task, we also evaluate our method on a large-scale short-term person Re-ID dataset: Market-1501. As shown in Table 4, our approach achieves comparable performance with short-term methods and far exceeds other long-term methods on Market-1501, which indicates the good generalization of our method.

Market-Clothes / PKU-Market-Reid. We use the model trained on the Market-1501 dataset to test on our two collected datasets. The experimental results are shown in the Table 5. On the challenging Market-Clothes dataset, other methods suffer significant performance declines, while ours remains stable and superior. On the real PKU-Market-Reid dataset, our method can still adapt to the complexity of realworld scenarios and achieve the best performance. These results can prove the domain adaptability of our method. The identity features clustering scatter plots are shown in Fig. 4.

\subsection{Ablation Study}

To investigate the effect of each component in our AFD-Net, we perform ablation studies on the Market-Clothes dataset.

Identity vs. clothing features. The best performance appears when identity and clothing features are concatenated as shown in Table 6 . To further show the independence of the two features, the feature visualization is performed on the Market-1501 dataset in Fig. 5. It shows that our AFD-Net can effectively disentangle the local clothing information and the global context-aware identity information of human body.

Intra-class vs. inter-class networks. We also compare the intra-class reconstruction and inter-class adversary networks as shown in Table 7 . It shows that the "intra+inter" surpasses "intra" and "inter" by a large margin, which indicates that the inter-class information is more important than the intra-class, and training the two networks end-to-end is effective.

Effect of re-feeding strategy. To evaluate the impact of the re-feeding strategy, we conduct an ablation study as shown in Table 8. Compared to "Non-Refeed", "Refeed" surpasses it by $+7.34 \%$ Rank-1 accuracy and $+2.51 \%$ mAP. It proves

\begin{tabular}{c|cc|cccc}
\hline Features & $s$ & $f$ & $\mathrm{R} 1$ & $\mathrm{R} 5$ & $\mathrm{R} 10$ & $\mathrm{mAP}$ \\
\hline Identity & $\checkmark$ & & 33.52 & 47.51 & 54.33 & 4.45 \\
Clothing & & $\checkmark$ & 38.24 & 55.34 & 62.74 & 6.32 \\
Identity+Clothing & $\checkmark$ & $\checkmark$ & $\mathbf{4 8 . 3 1}$ & $\mathbf{6 5 . 8 8}$ & $\mathbf{7 2 . 7 7}$ & $\mathbf{8 . 4 7}$ \\
\hline
\end{tabular}

Table 6: Ablation studies on different features.

\begin{tabular}{c|cc|cccc}
\hline Type & intra-class & inter-class & R1 & R5 & R10 & mAP \\
\hline Intra & $\checkmark$ & & 39.58 & 54.51 & 61.85 & 5.75 \\
Inter & & $\checkmark$ & 42.25 & 58.31 & 65.86 & 6.75 \\
Intra+Inter & $\checkmark$ & $\checkmark$ & $\mathbf{4 8 . 3 1}$ & $\mathbf{6 5 . 8 8}$ & $\mathbf{7 2 . 7 7}$ & $\mathbf{8 . 4 7}$ \\
\hline
\end{tabular}

Table 7: Ablation studies on different networks.

\begin{tabular}{c|c|cccc}
\hline Method & Re-feeding & R1 & R5 & R10 & mAP \\
\hline Non-Refeed & $\times$ & 40.97 & 55.43 & 62.35 & 5.96 \\
Refeed & $\checkmark$ & $\mathbf{4 8 . 3 1}$ & $\mathbf{6 5 . 8 8}$ & $\mathbf{7 2 . 7 7}$ & $\mathbf{8 . 4 7}$ \\
\hline
\end{tabular}

Table 8: Effectiveness evaluation of the re-feeding strategy.

\begin{tabular}{c|cc|cccc}
\hline Label & $y_{a}$ & $y_{b}$ & $\mathrm{R} 1$ & $\mathrm{R} 5$ & $\mathrm{R} 10$ & $\mathrm{mAP}$ \\
\hline$L_{a} L_{a}$ & $s, f$ & - & 40.83 & 57.04 & 65.29 & 6.60 \\
$L_{b} L_{b}$ & - & $s, f$ & 40.35 & 57.51 & 65.05 & 6.61 \\
$L_{b} L_{a}$ & $f$ & $s$ & 38.72 & 53.15 & 60.15 & 5.57 \\
$L_{a} L_{b}$ & $s$ & $f$ & $\mathbf{4 8 . 3 1}$ & $\mathbf{6 5 . 8 8}$ & $\mathbf{7 2 . 7 7}$ & $\mathbf{8 . 4 7}$ \\
\hline
\end{tabular}

Table 9: Effectiveness evaluation of the soft label strategy. As $x_{a b}$ for example, we show the different label selections for $s_{r e c}^{a b}$ and $f_{r e c}^{a b}$.

that the re-feeding part can effectively supervise the adversarial image generation, and further disentangle the identity and clothing features to make them more independent.

Effect of soft label strategy. We investigate how to set the supervised labels for disentangled features of generated images. As $x_{a b}$ for example, the comparisons are shown in Table 9. " $L_{a} L_{a}$ " and " $L_{b} L_{b}$ " denote that both features are set to the same label, whereas " $L_{b} L_{a}$ " and " $L_{a} L_{b}$ " denote different labels. Our soft label strategy " $L_{a} L_{b}$ " gets the excellent performance. This is because $s_{r e c}^{a b}$ and $f_{r e c}^{a b}$ are derive from $x_{a}$ and $x_{b}$, so setting their labels to $y_{a}$ and $y_{b}$ is effective.

\section{Conclusion}

In this paper, we propose an adversarial feature disentanglement network (AFD-Net), intending to address the clotheschanging problem in long-term person Re-ID. First, the AFDNet combines the intra-class reconstruction and inter-class adversary to disentangle the identity and clothing features, which can make these features more independent and discriminative. Second, AFD-Net can recombine the identity and clothing features across different identities to generate new reliable clothes-changing images for further disentangling. Third, a re-feeding strategy can supervise the adversarial image generation by image-level self-supervision in the original image space and feature-level soft-supervision in the disentangled feature space. Finally, to facilitate the research about the clothes-changing problem, we collect two longterm Re-ID datasets: Market-Clothes and PKU-Market-Reid.

\section{Acknowledgments}

This work is supported by National Natural Science Foundation of China (No.62073004), Science and Technology Plan of Shenzhen (No.JCYJ20190808182209321). 


\section{References}

[Chang et al., 2018] Xiaobin Chang, Timothy M. Hospedales, and Tao Xiang. Multi-level factorisation net for person re-identification. In ICCV, pages 2109-2118, 2018.

[Dai et al., 2017] Jifeng Dai, Haozhi Qi, Yuwen Xiong, Yi Li, Guodong Zhang, Han Hu, and Yichen Wei. Deformable convolutional networks. In ICCV, pages 764773, 2017.

[Ge et al., 2018] Yixiao Ge, Zhuowan Li, Haiyu Zhao, Guojun Yin, Shuai Yi, Xiaogang Wang, and Hongsheng Li. FD-GAN: pose-guided feature distilling GAN for robust person re-identification. In NeurIPS, pages 1230-1241, 2018.

[Geng et al., 2016] Mengyue Geng, Yaowei Wang, Tao Xiang, and Yonghong Tian. Deep transfer learning for person re-identification. CoRR, abs/1611.05244, 2016.

[Guo et al., 2019] Jianyuan Guo, Yuhui Yuan, Lang Huang, Chao Zhang, Jin-Ge Yao, and Kai Han. Beyond human parts: Dual part-aligned representations for person re-identification. In ICCV, pages 3641-3650, 2019.

[He et al., 2016] Kaiming He, Xiangyu Zhang, Shaoqing Ren, and Jian Sun. Deep residual learning for image recognition. In CVPR, pages 770-778, 2016.

[Huang et al., 2018] Xun Huang, MingYu Liu, Serge Belongie, and Jan Kautz. Multimodal unsupervised imageto-image translation. In ECCV, pages 179-196, 2018.

[Huang et al., 2020] Yan Huang, Jingsong Xu, Qiang Wu, Yi Zhong, Peng Zhang, and Zhaoxiang Zhang. Beyond scalar neuron: Adopting vector-neuron capsules for longterm person re-identification. TCSVT, 30(10):3459-3471, 2020.

[Jaderberg et al., 2015] Max Jaderberg, Karen Simonyan, Andrew Zisserman, and Koray Kavukcuoglu. Spatial transformer networks. In NeurIPS, pages 2017-2025, 2015.

[Ke et al., 2019] Bo Ke, Huicheng Zheng, Lvran Chen, Zhiwei Yan, and Ye Li. Multi-object tracking by joint detection and identification learning. Neural Process. Lett., 50(1):283-296, 2019.

[Krizhevsky et al., 2012] Alex Krizhevsky, Ilya Sutskever, and Geoffrey E. Hinton. Imagenet classification with deep convolutional neural networks. In NeurIPS, pages 11061114, 2012.

[Li et al., 2018] Wei Li, Xiatian Zhu, and Shaogang Gong. Harmonious attention network for person re-identification. In $I C C V$, pages 2285-2294, 2018.

[Li et al., 2020] Yu-Jhe Li, Zhengyi Luo, Xinshuo Weng, and Kris M. Kitani. Learning shape representations for clothing variations in person re-identification. CoRR, abs/2003.07340, 2020.

[Liu et al., 2018] Hao Liu, Zequn Jie, Jayashree Karlekar, Meibin Qi, Jianguo Jiang, Shuicheng Yan, and Jiashi Feng. Video-based person re-identification with accumulative motion context. TCSVT, 28(10):2788-2802, 2018.
[Qian et al., 2020] Xuelin Qian, Wenxuan Wang, Li Zhang, Fangrui Zhu, Yanwei Fu, Tao Xiang, Yu-Gang Jiang, and Xiangyang Xue. Long-term cloth-changing person reidentification. CoRR, abs/2005.12633, 2020.

[Shi et al., 2020] Wei Shi, Hong Liu, and Mengyuan Liu. Identity-sensitive loss guided and instance feature boosted deep embedding for person search. Neurocomputing, 415:1-14, 2020.

[Simonyan and Zisserman, 2015] Karen Simonyan and Andrew Zisserman. Very deep convolutional networks for large-scale image recognition. In ICLR, 2015.

[Suh et al., 2018] Yumin Suh, Jingdong Wang, Siyu Tang, Tao Mei, and Kyoung Mu Lee. Part-aligned bilinear representations for person re-identification. In $E C C V$, pages 418-437, 2018.

[Sun et al., 2018] Yifan Sun, Liang Zheng, Yi Yang, Qi Tian, and Shengjin Wang. Beyond part models: Person retrieval with refined part pooling (and A strong convolutional baseline). In ECCV, pages 501-518, 2018.

[Varior et al., 2016] Rahul Rama Varior, Mrinal Haloi, and Gang Wang. Gated siamese convolutional neural network architecture for human re-identification. In $E C C V$, pages 791-808, 2016.

[Wan et al., 2020] Fangbin Wan, Yang Wu, Xuelin Qian, Yixiong Chen, and Yanwei Fu. When person reidentification meets changing clothes. In CVPR, pages 830-831, 2020.

[Wang et al., 2018] Guanshuo Wang, Yufeng Yuan, Xiong Chen, Jiwei $\mathrm{Li}$, and $\mathrm{Xi}$ Zhou. Learning discriminative features with multiple granularities for person reidentification. In ACMM, pages 274-282, 2018.

[Yang et al., 2020] Qize Yang, Ancong Wu, and Wei-Shi Zheng. Person re-identification by contour sketch under moderate clothing change. CoRR, abs/2002.02295, 2020.

[Yu et al., 2017] Qian Yu, Xiaobin Chang, Yi-Zhe Song, Tao Xiang, and Timothy M. Hospedales. The devil is in the middle: Exploiting mid-level representations for crossdomain instance matching. CoRR, abs/1711.08106, 2017.

[Yu et al., 2020] Shijie Yu, Shihua Li, Dapeng Chen, Rui Zhao, Junjie Yan, and Yu Qiao. Cocas: A large-scale clothes changing person dataset for re-identification. In CVPR, pages 3400-3409, 2020.

[Zheng et al., 2015] Liang Zheng, Liyue Shen, Lu Tian, Shengjin Wang, Jingdong Wang, and Qi Tian. Scalable person re-identification: A benchmark. In ICCV, pages 1116-1124, 2015.

[Zheng et al., 2017] Liang Zheng, Hengheng Zhang, Shaoyan Sun, Manmohan Chandraker, Yi Yang, and Qi Tian. Person re-identification in the wild. In $C V P R$, pages 3346-3355, 2017.

[Zheng et al., 2019] Zhedong Zheng, Xiaodong Yang, Zhiding Yu, Liang Zheng, Yi Yang, and Jan Kautz. Joint discriminative and generative learning for person reidentification. In CVPR, pages 2138-2147, 2019. 